\title{
EFFECTS OF CHINESE FONT STYLE AND COLOR ON VARIABLE MESSAGE SIGNS
}

\author{
Chien-Jung Lai, Kuo-Duan Yen, Duan-Bing Wang \\ Department of Distribution Management \\ National Chin-Yi University of Technology \\ Taiwan, ROC \\ E-mail: laicj@ncut.edu.tw
}

\begin{abstract}
Summary: Variable message signs (VMS) are important traffic control devices for increasing road utility and decreasing traffic accidents. This study investigated the effects of Chinese font style (Hei, Ming, and Kai) and font color (red, green, and yellow) of VMS on subjects' response performance (response time and accuracy) from an ergonomic viewpoint. Computer-generated VMS stimuli were merged with driver's-view driving video and projected onto a screen while subjects made responses. The results showed that Chinese font style and font color both were significant factors in subjects' response performance. Subjects took less response time for Hei style than for Kai and Ming styles, and exhibited higher accuracy for Hei and Ming styles than for Kai style. Subjects responded faster and more correctly for yellow and green font colors than for red. Additionally, the interaction of font style and font color had a significant effect on subjects' response performance. Response times for yellow and green colors were shorter than for red on each font style. Subjects had the longest time for Ming style in red color, while they had the least correct for Kai style in red color. The results from this study may assist in adopting appropriate Chinese font and color on VMS and in improving safe and efficient driving for motorists on the freeway.
\end{abstract}

\section{INTRODUCTION}

Variable message signs are programmable traffic control devices that display dynamic messages composed of letters, symbols, or both, about road information. The information displayed on VMS can come from a variety of traffic monitoring and surveillance systems. They can be changed by a system monitor through remote control or automatic controls that can sense the conditions requiring special messages. With more sophisticated technologies, VMS are gaining widespread use in intelligent transportation systems (ITS). They are critical for informing motorists of various situations, especially in high-volume traffic and congested zones. Due to the quick development of electronic technology and the increasing need to convey more information to motorists, variable message signs have been widely implemented on freeways recently in Taiwan.

Many investigations have been performed on evaluating the effects of VMS on drivers' response and behavior. A survey of more than 500 motorists in the Washington, DC, area assessed motorists' attitudes toward VMS and the effect of demographic characteristics on these attitudes (Benson, 1996). In the responses to the survey question regarding how often VMS influenced their driving, half the respondents replied "often," 40\% answered "occasionally," and the others indicated "not at all." It was also found that demographic variables, such as age, income and gender, appeared to have little influence on motorist attitudes about VMS. The interview surveys 
conducted by Chatterjee, et al. (2002) in London revealed that $97 \%$ of drivers were aware of the existence of VMS, $62 \%$ completely understood the information presented on VMS, $84 \%$ considered the information presented to be useful, and $46 \%$ had on at least one occasion diverted in response to the travel time information. The research on travelers' response to VMS in Paris concluded that VMS alone could affect vehicle diversion significantly. VMS were most effective when displayed during periods of increasing congestion, and the responses to VMS were more significant during morning peak hours than evening peak hours; the longer the queue length posted in VMS, the more vehicles diverted (Yim and Ygnance, 1996).

Many factors can affect the effectiveness in the design and display of VMS. Proper selection of factors and levels are very important. Armstrong and Upchurch (1994) conducted a field study in Phoenix, Arizona, on human factors design considerations such as legibility distance, target value, and viewing comfort for fiber-optic and LED VMS on freeways. They concluded that the optimum number of words contained in a message varied with the VMS technology, the lighting conditions, and the prevailing traffic speed. For VMS using other technologies or with different character fonts or dimensions, a legibility analysis prior to its implementation should be performed. Wang and Cao (2003) conducted a simulated study to evaluate the display formats of variable message signs. Real video scenes were integrated with computer-generated VMS to create a simulated driving environment. They found that the number of font colors, message lines, driving lane, and display format all significantly affected response time. The results showed that a green font color resulted in the least response time, while a red color resulted in the longest.

Most of the investigations of variables concerning information presentation on VMS have been done within the context of Western language alphabets. The results of some of this research may not be generalized to Chinese VMS information presentation because of significant differences between printed English and Chinese characters, as well as differences between the conventional methods of arranging printed material and the reading patterns typical of Western and Chinese cultures. Chinese characters have a completely different structure and appearance. Every Chinese character is a word that has its own meaning and unique appearance. Therefore, reading Chinese characters is a kind of symbol recognition process, whereas recognizing English words is a "spelling" process (Hwang, Wang \& Her, 1988). Because of the completely different recognition process of English and Chinese, the results obtained from studies of English information displays on VMS may not be applicable to Chinese VMS information display. Therefore, the major objective of this study was to investigate the effects of Chinese font style and font color of VMS on subjects' response performance.

\section{METHOD}

\section{Subjects}

Twenty-four college students (12 female and 12 male) between 18 and 30 years old $(M=24$, $S D=2.14$ ) participated in the experiment. All had 0.8 corrected visual acuity or better and normal color vision. Each subject had a driver's license and experiences driving on the freeway. They were paid for their participation. 


\section{Stimuli and Apparatus}

The stimuli used in this study were composed of a sequence of computer-generated VMS merged with a real drivers' view video representing freeway driving at $100 \mathrm{~km}$ per hour. Four messages were displayed on the VMS concurrently. Each stimulus used black as the background color and had a different combination of font style and font color. Table 1 shows the contents of four messages on VMS and their associated response mode via wheel and pedal.

Table 1. The four messages on VMS and associated response mode

\begin{tabular}{ll}
\hline Message (in Chinese) & Associated response mode \\
\hline Slow down to pay toll & Press left pedal \\
Accident removed, speed up to pass & Press right pedal \\
Right lane closed, change to Left & Turn the wheel to left \\
Left lane closed, change to right & Turn the wheel to right \\
\hline
\end{tabular}

A Topcon Screenscope SS-3 was used to test subjects' visual acuity and color vision. The CIE color values were measured with a Minolta color analyzer CA-100. A P4-2.4 PC with 1G ram was used to process the experimental task program to the subjects. A Microsoft side winder force feedback wheel, replacing the steering wheel, was installed in front of the driver's seat of a refitted used car in the ergonomic laboratory. The stimuli were projected on a screen $(290 \mathrm{~cm}$ wide $\times 200 \mathrm{~cm}$ high) through an EPSON EMP-730 projector.

\section{Experimental Design}

The independent variables were the Chinese font style and font color. There were three styles of font: Hei, Kai, and Ming. They are all widely used in Chinese textual displays. Illustrations of the three font types are shown in Table 2. Red, green, and yellow were used for the variable of font color. A within-subjects design was conducted. Nine treatment groups represented the combinations of the two within-subjects factors of font style and font color. Each subject went through a total of 72 randomized VMS presentations, i.e., 36 different VMS $(3$ styles $\times 3$ colors $\times 4$ messages) with 2 repetitions in each test block. A subject was tested on two successive test blocks.

Table 2. Illustrations of the three font styles used in this study

\begin{tabular}{ll}
\hline Font style & Example \\
\hline Hei & 右側封閉 靠左行駛 \\
Kai & 石側封閉 靠左行弱 \\
Ming & 右側封閉 啡左行駛 \\
\hline
\end{tabular}

\section{Conditions of Workplace}

To simulate a freeway-driving environment, computer-generated VMS were merged with a driver's view video and projected onto a screen in front of the test vehicle. Figure 1 shows the experimental task configuration. The distance between subject and screen was $700 \mathrm{~cm}$. The 
ambient illumination was about 300 lx, tested at the subject's seat, and $500 \mathrm{~lx}$ at the screen.

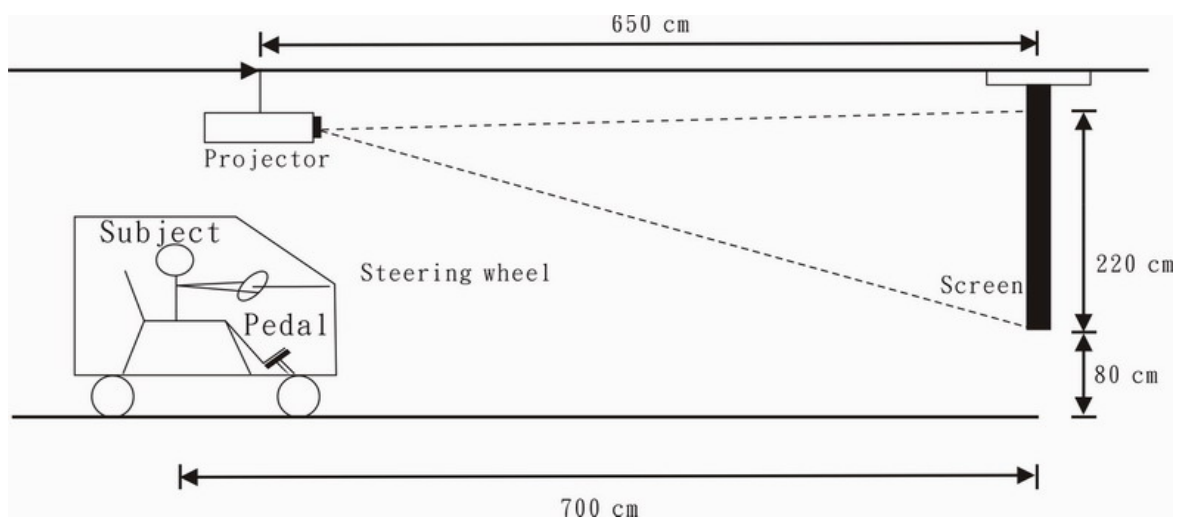

Figure 1. The experimental task configuration

\section{Task and Procedure}

A test subject, sitting in the driver's seat of a refitted used car, would see the driving video on the screen with VMS stimuli appearing on the road center and gradually increasing in size. The time interval allowed for each VMS presentation was 10 seconds; presentation would terminate when a response was made. After a response was made and the VMS stimuli disappeared, the next stimuli would appear after a random elapse of between 1 and 3 seconds. Each subject was instructed to identify the message presented during each trial by turning the steering wheel by hand or pressing the pedal by foot to signify her/his comprehension of a specific message. Subjects' response time and accuracy were recorded by the computer automatically.

Before starting the experiment, each subject was briefed on the purpose and procedures of the experiment. In order to prevent visual fatigue, subjects were asked not to do any reading tasks for an hour before the experiment. A warm-up session was conducted in the beginning to familiarize participants with the response task. With the subjects' consent, the actual experiment started. The complete experiment took about one hour.

\section{Performance Measure and Data Analysis}

Response time and correct percentage were collected in this experiment. Response time was the time between the presentation of a VMS stimulus and the subjects' correct response to the message. Correct percentage was 100 times the number of VMS correct responses divided by the total number of VMS. Analysis of variances (ANOVA) was conducted on the dependent measures. The factors that were significant were further analyzed using Tukey grouping method to discuss the difference among the factor levels. All statistical analyses were made with the Statistical Analysis System (SAS).

\section{RESULTS}

\section{Response Time}

The results of the analysis of variances on font style and font color showed that the main effect 
of font style was significant $(\mathrm{F}(2,46)=162.15, \mathrm{p}<0.01)$. While the font styles were Hei, Ming, and Kai, the mean response time was $4.7618,5.3503$, and $5.1713 \mathrm{sec}$, respectively. The results of Tukey multiple-test demonstrated that Hei style was significantly different from Kai and Ming styles.

The results of the analysis of variances also showed that the main effect of font color $(\mathrm{F}(2,46)=544.93, \mathrm{p}<0.01)$ was significant. The mean response times were 5.8022, 4.8037, and $4.6775 \mathrm{sec}$, respectively, while the font colors were red, green, and yellow. The results of Tukey multiple-test demonstrated that the font color group of yellow and green was significantly different from that of red.

The results of the analysis of variances showed that the interaction of font type and font color had a significant effect on response time $(F(4,92)=3.77, p<0.01)$. Figure 2 shows that response times for green and yellow were shorter than for red in each font style. Subjects took the shortest time for Hei style in yellow and green, while they took the longest time for Ming style in red.

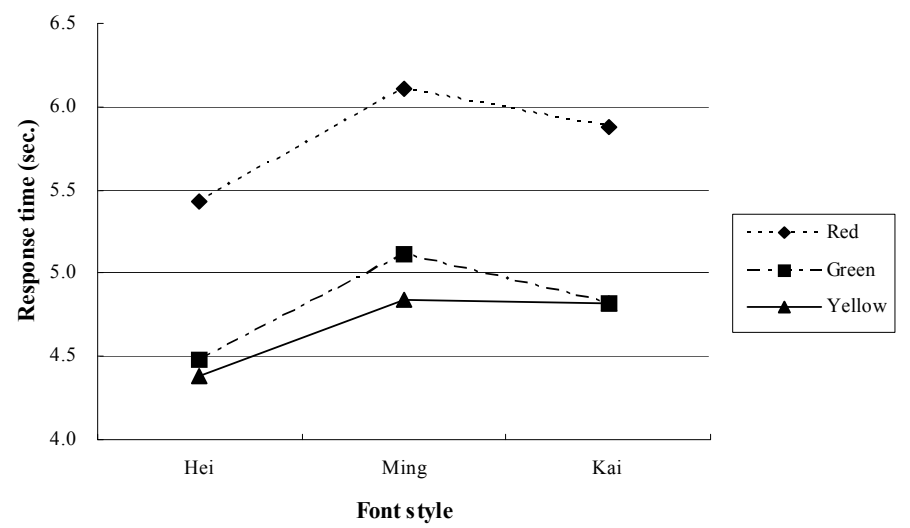

Figure 2. Interaction of font style and font color on response time

\section{Correct Percentage for Font Style and Font Color}

Analysis of variance performed on correct percentages also found that font style $(\mathrm{F}(2$, $46)=119.19, \mathrm{p}<0.01)$, font color $(\mathrm{F}(2,46)=67.62, \mathrm{p}<0.01)$, and their interaction $(\mathrm{F}(4,92)=49.87$, $\mathrm{p}<0.01)$ were all significant. Subjects had fewer correct responses $(87.67 \%)$ for Kai style than for Hei style (96.87\%) and Ming style (96.27\%). The results of Tukey multiple-test demonstrated that the Kai font style was significantly different from the Hei and Ming styles. While the font colors were red, green, and yellow, the mean correct percentages were 87.58, 96.35, and 96.87 respectively. The results of Tukey multiple-test also showed that the font color groups of green and yellow were significantly different from that of red. Figure 3 shows the interaction of font style and font color on correct percentage. Subjects' responses were least correct for the Kai style in red. 


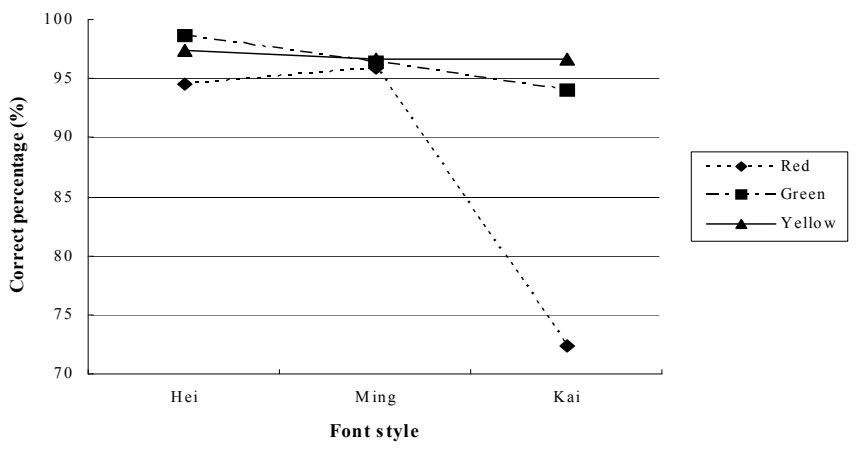

Figure 3. Interaction of font style and font color on correct percentage

\section{DISCUSSION}

\section{Font Style}

The results of this study showed that font style affected subjects' performance significantly in responding to VMS messages. Subjects responded faster for Hei style than for Kai and Ming styles. They also had more correct responses for Hei and Ming styles than for Kai style. This result is similar to the findings of a survey conducted by Lai and Yen (2004) in Taiwan. In their questionnaire survey, drivers preferred Hei (43.6\%) and Kai (43.9\%) styles to Ming (12.5\%). The differences between the performance measures of the three font styles could possibly be explained by the characteristics of the characters. Compared to Ming and Kai styles, Hei style is of uniform stroke width and has no serifs. The characteristics of Hei style are similar to that of Gothic letters in English. The vertical and horizontal strokes are all the same width (Cai et al., 2001; Qiu, 1991). For Ming and Kai, the width is not all the same. In Taiwan, Hei style is the most common Chinese style used in fixed traffic signs. This study supported that the Hei style may also have benefits for variable message signs.

\section{Font Color}

In this study, font color also affected subjects' response performance significantly. Subjects had faster and more correct responses for yellow and green fonts than for red. This result is similar to the finding of the research conducted by Wang and Cao (2003) in Rhode Island. Their research of English VMS also found that subjects had longer response times for red fonts than for green and yellow fonts. However, this is different from the survey conducted by Lai and Yen (2004) at the rest area on freeway in Taiwan. Their questionnaire survey showed that drivers preferred yellow (42.6\%) and red (40.1\%) font colors to green $(17.3 \%)$. The difference may be caused by the driver's experience of the colors. In Taiwan, the font colors of VMS on freeway are almost always yellow and red. The frequent contact with existing VMS could account for the drivers' preference of yellow and red.

\section{Interaction of Style and Color}

The result of the analysis of variance also showed that the interaction of font style and font color had a significant effect on subjects' response time and accuracy. Response times for green and 
yellow colors were shorter than for red in each font style. Subjects took the longest time for the Ming style in red, and they had fewest correct responses for Kai style in red. Considering the main effect of font style and font color together, the combination of Hei style in yellow, or Hei style in green could be the better choice for the Chinese VMS display.

\section{CONCLUSIONS}

The major objective of this study was to investigate the effects of Chinese font style and font color on variable message signs display. The study showed that font style, font color and their interaction all had significant effects on subjects' response time and accuracy. Subject responded faster for Hei style than for Kai and Ming styles. The percentage of correct responses was greater for Hei and Ming styles than for Kai style. Subjects also responded faster and more correctly to yellow and green than to red in each font style. In conclusion, Hei style in yellow or green could be better for Chinese VMS display. However, the results of this experimental study for font color are different from another questionnaire survey, and further studies should be considered.

\section{ACKNOWLEDGMENTS}

This study was supported by a Research Grant from the National Science Council of the Republic of China, Grant no. NSC 93-2213-E167-015.

\section{REFERENCES}

Armstrong, J.D., Upchurch, J.E. (1994). Human factors design considerations for variable message freeway signs. Journal of Transportation Engineering, 120(2), 264-282.

Benson, B.G. (1996). Motorist attitude about content of variable message signs. Transportation Research Record, 1550, 48-57.

Cai, D., Chi, C.F., and You, M. (2001). The legibility of Chinese characters in three-type styles. International Journal of Industrial Ergonomics, 27, 9-17.

Chatterjee, K., Hounsell, N.B., Firmin, P.E., Bonsall, P.W. (2002). Driver response to variable message sign information in London. Transportation Research Part C, 10(2), 149-169.

Hwang, S.L., Wang, M.Y., Her, C.C. (1988). An experimental study of Chinese information display on VDTs. Human Factors, 30(4), 461-471.

Lai, C.J., Yen, K.T. (2004). Sedan drivers' attention and response to variable message signs on freeway in Taiwan. The 4th International Conference on Traffic and Transportation Psychology, Nottingham, UK.

Qiu, Y.F. (1991). Typography: The theory and practice of type styles. Taipei: Yi Feng Tang Publishing Co, (in Chinese).

Wang, J.H., Cao, Y. (2003). A human factors study on message design of variable message sign. International Journal of Industrial Engineering, 10(4), 339-344.

Yim, Y., Ygnance, J.L. (1996). Link flow evaluation using loop detector data: traveler response to variable message signs. Transportation Research Record, 1550, 58-64. 